\title{
An Update on the Role of Bone Morphogenetic Protein and Growth Differentiation Factor on Follicular Generation in Human Menstrual Cycle with Clinical Impact on ART Success and PCOS Pathogenesis/ Therapy-a Systematic Review
}

\author{
Kulvinder Kochar Kaur ${ }^{1 *}$, Gautam Allahbadia ${ }^{2}$ and Mandeep Singh ${ }^{3}$ \\ ${ }^{1}$ Scientific Director, Dr Kulvinder Kaur Centre for Human Reproduction, Jalandhar, \\ Punjab, India \\ ${ }^{2}$ Scientific Director, Ex-Rotunda-A Centre for Human Reproduction, Mumbai, India \\ ${ }^{3}$ Consultant Neurologist, Swami Satyanand Hospital, Jalandhar, Punjab, India \\ *Corresponding Author: Kulvinder Kochar Kaur, Scientific Director, Dr Kulvinder \\ Kaur Centre for Human Reproduction, Jalandhar, Punjab, India.
}

Received: January 21, 2022

Published: January 31, 2022

(C) All rights are reserved by Kulvinder

Kochar Kaur., et al.

\begin{abstract}
Background: With the initial identification of capacity of generation of heterotopic bone Bone morphogenetic proteins (BMP) represent growth factors that possess numerous functions from the transforming growth factor beta (TGF- $\beta$ ) super family, With the utilization of cellular along with molecular genetic approaches, more recently studies have implied that that intraovarian BMP 's work as robust functional controllers of ovarian follicular function. The crosstalk that takes place in a 'bidirectional manner of the oocytes besides the somatic cells surrounding them is a must for the normal follicular generation along with maturation of the oocytes. Thus we reviewed the existent information regarding the physiological part as well as the molecular determining factors of these ovarian controlling factors amongst the germline somatic -controlling loop.

Aim: The control of ovarian function has been ill characterized amongst humans, as whereas the elemental event of follicular generation along with maturation of the oocytes is akin in different species, with maximum knowledge in the context of control of ovarian follicular function gets derived via rodent studies. Hence the concentration was on studies where utilization of human biological materials done for getting insight in the context of human ovarian biology for the generation of strategies with the aim of avoidance, diagnosis therapy treatment, diagnosis besides treatment of these aberrations.

Methods and Results: The outcomes studies with utilization of human biological materials documented the expression of BMP 's or GDF's in addition to their respective receptors along with their molecular signaling in the basic cells (oocytes, cumulus/granulosa cells (GCs) besides theca/stroma cells) of the ovarian follicles right through generation. On the existence of recombinant BMP 's/ GDF's along with generation the physiological functional part of human intraovarian BMP 's/GDF's have been illustrated in each aspect of function of ovary ranging from i) follicle generation ii) steroidogenesis iicell-cell contact, iv) maturation of the oocytes v) ovulation and luteal function. Moreover, crosstalk amongst these ovarian controllers and endocrine signaling system. Impairment or natural mutations amongst the BMP system might result in numerous female reproductive diseases. With the generation of recombinant BMP, synthetic BMP inhibitors, gene therapy, tools meant for BMP ligand concealment, has seen to it that the BMP pathway has become a probable therapeutic target in some fertility pathological disorders. Nevertheless, further clinical trials are required. More recently GDF 8 has been demonstrated to be an intraovarian factor that might play an innovative part in control of ovarian functions in human ovary.
\end{abstract}

Keywords: Bone Morphogenetic; Follicular Generation; Ovulation; PCOS; Physiological; Endocrine

Citation: Kulvinder Kochar Kaur., et al. "An Update on the Role of Bone Morphogenetic Protein and Growth Differentiation Factor on Follicular Generation in Human Menstrual Cycle with Clinical Impact on ART Success and PCOS Pathogenesis/Therapy-a Systematic Review". Acta Scientific Women's Health 4.2 (2022): 58-72. 
An Update on the Role of Bone Morphogenetic Protein and Growth Differentiation Factor on Follicular Generation in Human Menstrual Cycle with Clinical Impact on ART Success and PCOS Pathogenesis/Therapy-a Systematic Review

\section{Introduction}

In humans the ovarian follicle represents the basic functional unit with regards to the female reproductive system, that might be parallel to the various generation stages in the ovary. At the time of the female reproductive cycle the propagation of these follicles occurs via a tightly synchronized controlled event where numerous neural, neuroendocrine along with autocrine/paracrine regulation systems are implicated for attainment of total ovulatory in addition to steroidogenic capacity. The initial part of the pituitary hormones, gonadotrophins, (FSH, Luteinizing hormone ( $\mathrm{LH})$, gonadotropin releasing hormone ( $\mathrm{GnRH})$, gonadal hormones (estrogen - androgens, progesterone) in the female reproductive system, have been well corroborated. Nevertheless, normal follicular function along with follicular generation is based on numerous locally generated growth factor $\mathrm{s}$ in addition to cytokines whose influence is exerted via their actions in an autocrine, paracrine manner [knight Glister 06]. Over the last few decades it has been illustrated in numerous species that are members of the transforming growth factor beta (TGF- $\beta$ ) superfamily, that are inclusive of the Bone morphogenetic proteins (BMP), the growth differentiation factors (GDFs), TGF- $\beta$, activin inhibin's besides antimullerian hormone (AMH) get expression in the ovary possessing necessary part in the control of folliculogenesis, oogenesis along with ovarian functions [1]. Constituted by greater than 20 members BMP/GDFs comprise the biggest subfamily of the TGF- $\beta$ superfamily, getting isolated earlier in the form of osteo inducing cytokines that were implicated in the facilitation of bone along with cartilage generation [2]. Various published articles have revealed that locally generated BMPs participate in the generation along with function of mammalian germ cells, besides them correlated with control of follicular ovarian functions [3]. Furthermore, natural mutations amongst the BMP ligands in addition to impairment of BMP signaling have been correlated with significant pathologies of human reproduction [4].

Whereas the foundational event of follicular generation, is remarkably akin to each other in numerous species, maximum knowledge on the controlling of ovarian functions has been derived from rodent studies. The insight we have generated in the context of generation along with function of the human ovary advancements has occurred just more recently in view of the combination of technologies that have been inclusive of recombinant human BMP/GDFs, of immortalized human granulosa cells (GCs) lines, tissue microarray along with, pharmaceutical generation.
BMPs as well as BMP receptors

\section{Ligands of BMP}

BMPs comprise of a large subfamily of growth factors that belong to the TGF- $\beta$ ) superfamily have got evolutionary preserved that possess key functions in the context of organogenesis, along with morphogenesis in case of both invertebrates as well as vertebrates (rev in 6). With regards to the modern classification, BMP s are considered as the surrounding growth factors which might be BMP or GDFs having the knowledge of overlap amongst the 2 classification systems without any important different lineages $[5,6]$ (Figure1). Just akin to the other members of TGF- $\beta$, superfamily BMP s originally undergo translational alterations in the form that possess akin structure that gets constituted of 3 parts namely signal peptide, that is a prodomain along with a mature peptide (mature domain), The signal peptide made up of 20-30 amino acids, is existent at the $\mathrm{N}$ terminal end of the generated fresh pre proteins, that guides these ligands for the appropriate liberation out of the cells [7]. A conserved structure, like the seven cysteine residues whose spacing is done with the possession of appropriate characteristics which point to a cysteine knot motif of the TGF- $\beta$ superfamily. 6 of these cysteine residues possess the capacity of generation of intra molecular disulfide bond that are labelled as cysteine knot, with the 7th cysteine being implicated in the dimerization with a different monomer through a disulfide bond to develop homodimers or heterodimers [8]. Nevertheless, BMP15 as well as GDF9 are 2 of the rare TGF- $\beta$, family members that are implicated in the covalent dimer generation [8]. Proteolytic cleavage of the inactive precursors of BMP takes place by the utilization of proproteins convertase family; preproteins convertase Subtilism/Kexin that comprises of 9 members known as PCSK1- PCSK [9]. A minimum of one member that belong to this family PCSK3 (alias furin), possesses the capacity of recapitulation the amino acids motif -Arg-X-Arg/Lys- Arg -at the linker region amongst the prodomain along with a mature domain in addition to resulting in the activation of the mature BMP homodimers or heterodimers [10]. Various BMP heterodimers like BMP2/BMP7, BMP2/BMP6, BMP4/BMP7 known as, BMP15 along with GDF 19 have been pointed to be existent besides work in vivo along with in vitro [11]. Maximum of these BMP heterodimers possess greater biological robustness in the induction of cellular functions [11]. The biggest reason for these differential actions is that the heterodimer action might get modulated via a separate receptor kind or an extra receptor sub kind. There has been a wide de- 
An Update on the Role of Bone Morphogenetic Protein and Growth Differentiation Factor on Follicular Generation in Human Menstrual Cycle with Clinical Impact on ART Success and PCOS Pathogenesis/Therapy-a Systematic Review

bate with regards to the biological effect of oocyte obtained BMP15 as well as GDF 9 is dispensable, or synergism exists. A study demonstrated that Bmp 15 as well as Gdf 9 double knockout mice illustrated that a biological harmony amongst these 2 growth factors is existent. A large altercation with regards to if this coordination occurs secondary to a synergistic crosstalk of BMP15 as well as GDF 9 homodimers or from the biologically present BMP1/GDF 9heterodimer. Intriguingly the BMP1/GDF 9heterodimer [11,12] generation as well as liberation can take place in the $293 \mathrm{~T}$ cells (a particular cell line that was initially obtained from human embryonic kidney cells) when co- expression of the 2 constructs occurs. Actually, the human, BMP15/GDF 9 heterodimer possesses about 1000-3000 times greater potency in contrast to the human BMP15 homodimer [11].

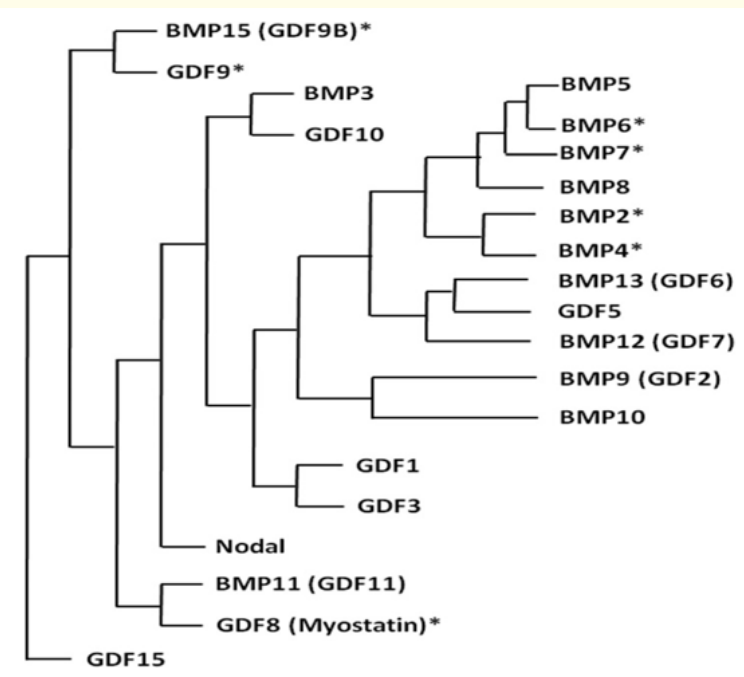

Figure 1: Courtesy ref no-6- Phylogenetic relationship of the BMP/GDF subfamily members. The alignment of $21 \mathrm{BMP} /$ GDF-related ligands was based on published literature. Ligands with an asterisk are expressed in the human ovary. BMP/GDF, bone morphogenetic protein/growth differentiation factor.

\section{BMP receptors}

The signaling of TGF- $\beta$ superfamily members implicates various set of trans membrane serine/threonine kinase receptors (Type1 as well as 2 receptors) that further control downstream gene expression by phosphorylation of the Sma as well as Mad -related protein (SMAD) Transcription factors. Till date 5 separate Type II receptors (BMP R2, ACV R 2A, ACV R 2B, T $\beta$ R2 as well as AMHR2) along with 7 type 1 receptors (alias activin receptor like kinase, ALK1-7 have got isolated in mammals, there is a common sharing of akin structure comprised of an extracellular domain ( $\mathrm{N}$ terminal), a trans membrane domain in addition to an intracellular domain with Ser/Thr kinase action ( $C$ terminal) by all of these receptors. Every ligand of the TGF- $\beta$ super family members possess, the capacity of binding to the $\mathrm{N}$ terminal extracellular domain of various combinations of Type II as well as Type I receptors besides resulting in downstream signaling complexes [6]. Of the 5 Types I receptors, three (BMP R2, ACV R 2A, ACV R 2B) utilization is done by 1 or greater BMPs. Similarly, six (ALK2-7) of the seven have been involved in BMP stimulated signaling [13].

\section{BMP signaling pathway}

TGF- $\beta$ superfamily members Influence their action by binding to type I as well as Type II receptors, that further control downstream gene expression via phosphorylation of the receptor-controlling SMAD (R- SMAD) transcription factors, SMAD. Usually, 2 separate models of the ligand -receptor crosstalk is present. SMAD 1/5/8 activation through ALK2, ALK3 along with ALK6 occurs by BMPs, AMH as well as certain GDFs, while TGF- $\beta$ s, activin besides nodal results in activation of SMAD 2/3 through ALK4, ALK5 along with/or ALK7[14]. On phosphorylation of type I receptors, the activation of the accepted SMAD 1/5/8 group then correlates with the usual SMAD (SMAD4), with the translocation towards the nucleus to influence the functions which control gene expression by maximum tissues.

More recently, innovative nontraditional SMAD 2/3 signaling (whose induction (is usually done by TGF- $\beta$ along with activins) activation by the BMPs for control of cancer propagation along with hormone generation has received marked importance [15]. In case of human granulosa tumor cells in addition to mouse GCs, recombinant human BMP 15, however not mouse BMP 15resulted in mild phosphorylation of SMAD 2/3, besides stimulation of mouse cumulus oocyte complex (COC) expansion [11]. In agreement with this documentation which have queried the standard guidelines that BMPs influence cellular functions just alone via the traditional SMAD 1/5/8 signaling, the latest studies by Chang's group demonstrated that BMPs result in hyaluronan synthase type 2 (HSA2) expression was modulated by the nontraditional SMAD 
An Update on the Role of Bone Morphogenetic Protein and Growth Differentiation Factor on Follicular Generation in Human Menstrual Cycle with Clinical Impact on ART Success and PCOS Pathogenesis/Therapy-a Systematic Review

2/3 signaling via binding to ALK4, ALK5/ALK7 in the of immortalized human GCs [16]. Variations in receptor expression amounts as well as receptor binding characteristics, amongst, various BMPs (both the homodimer and. heterodimer) probably possess the biggest chance of differential effects in nontraditional SMAD 2/3 signaling in certain cell kinds. Besides SMAD signaling, various SMAD independent (non SMAD) signaling pathway for BMPs have been isolated in particular tissues. It has been strongly thought that the mitogen activated protein kinase (MAPK) signaling pathway is the most significant SMAD independent pathway that gets stimulated by BMPs. BMP 4 has been seen to result in phosphorylation of TGF- $\beta$ activated kinase1, that is a serine/threonine kinase belonging to the MAPKKK family [6]. Earlier studies pointed that BMP $s$ further possess the capacity of activation of various signaling pathway, that is inclusive of extracellular signal -regulated kinase (ERK1/2), protein kinase (PK) PK A, PK C, as well as PK D (PK C $\mu$ ) [17]. Intriguingly the most recent study by Fang et al. [18] in 2015 demonstrated that the BMP subfamily member GDF8 resulted in reduction of steroidogenic acute regulatory protein (StAR) expression via activation, of SMAD 3 as well as ERK signaling pathway [18]. Once ligand receptor -binding as well as crosstalk occurred the following pathway activation, by BMP occurred most probably based on other cellular action, the extracellular milieu in addition to interaction with other signaling pathways.

\section{Intraovarian BMPs as well as oocyte -somatic cell crosstalk}

Human granulosa cells (GCs) lines

For getting a better insight on modes by which hormones or growth factors control folliculogenesis as well as ovarian steroidogenesis, cell models that are existent along with appropriate with regards to in vitro studies are desired. GC dependent in vitro model systems have yielded promising methods for evaluation of ovarian biology. The biggest source of human GCs with regards to in vitro studies comes most commonly from infertility patients that are getting invitro fertilization (IVF) treatment. Nevertheless, these cells can be readily obtained just in small amount, making it tough to carry out larger experiments that are correlated with molecular approaches in detail. Additionally, the clinically derived GCs are mostly luteinized in view of them getting exaggerated stimulation with the utilization of FSH/LH as well as HCG before the cells get isolated. Hence these GCs possess restricted life, besides the proliferation rates are slower. Thus, their survival in vitro is restricted with regards to multiple passages. Moreover, primary GCs obtained from separate patients might illustrate variations, that sums up the tough hurdles encountered in reproducting these outcomes. In view of these difficulties, a substituted human luteinized granulosa cell (GCs) lines have assumed a promising alternative. In the last decade there has been escalation of attention with regards to the generation of various cell lines from human GCs. These cell lines got developed via different methods that were inclusive of oncogenic transformation [6], transfection with site directed mutagenesis [19] as well as explants of human tumors. Noticeably, every cell lines might possess, separate characteristics in the context of steroidogenic functions, response to various gonadotrophins, (FSH as well as LH) cAMP responsiveness, BMP responsiveness, along with mitogenic in addition to differential capacities [6]. Hence it has become necessary of choosing the proper cell line for separate studies. Like the SVOG cell line generation was done by the transfection of human granulose-lutein (hGL) cells, utilization of SV 40Tcell antigen. Utilization of primary hGL) cells was done for development of immortalized cells besides the 2 illustrate akin responses biologically towards separate therapies, like LH, HCG, cAMP along with different growth factors. The laboratory of Chang., et al. has illustrated in various earlier publications in addition to other workers in the context of these cell being broadly agreed model with regards to studying the ovarian response at the time of periovulatory along with early luteal phases [20-24]. COV434 along with KGN are the 2 most commonly utilized GC cell lines that are initiated from ovarian GC tumors. To our misfortune the generation, of an androgen generating theca cell line has gone through difficult hurdles with restricted success [6].

\section{BMP as well as BMP receptors expression in human ovary}

Numerous studies that have been conducted have yielded significant knowledge in the context of expression of the BMP system in the mammalian ovary in the last decade with the utilization of maximum detailed study in adult cycling rats [6]. Nevertheless, the expression manner of BMPs in rat ovary might not be applicable in other species. Like BMP4 as well as BMP7 primary expression takes place in the theca as well as stroma cells in rat ovary, however BMP4 as well as BMP7 have been found in the human oocytes [6]. Identification, of GDF9 has been done in hGL cells as well as oocytes [6], however GDF9 expression takes place only in the oocytes of ovine as well as bovine ovaries. Parallel to that BMP15, that was 
An Update on the Role of Bone Morphogenetic Protein and Growth Differentiation Factor on Follicular Generation in Human Menstrual Cycle with Clinical Impact on ART Success and PCOS Pathogenesis/Therapy-a Systematic Review

earlier believed to get obtained from oocytes, has been observed in the human cumulus cells [25], GCs as well as stroma cells in girls along with adults. The generation in addition to the physiological functions of the human reproductive system get impacted maximum by the tissue particular along with time - based expression of BMP sub family members, [6] despite the spatiotemporal expression of BMPs in human ovary are not clear at large. In the human ovary expression of BMP2, BMP4, as well as BMP6, BMP7 along with BMP8A occurs in the GCs from the normal, cycling in addition to Polycystic ovary syndrome (PCOS) women $[25,26]$. In coordination with this study, the utilization of preantral in a study that contrasted the expression amounts of BMP in five size matched populations illustrated that BMP4 as well as BMP6 ligands [27]. In case of human corpus luteum, there is abundant expression of BMP2, B MP4 along with BMP6, in the granulosa -lutein along with theca lutein cells besides being implicated in the eventof luteolysis [28].

At the time of antral follicular stage, the growth along with maturation of the oocyte are based on various intraovarian factors, that are existent in follicular fluid [6]. The amounts of certain of the follicular fluid growth factors are associated with the serum amounts of these factors [11]. Aberrations of follicular generation besides impairment of oocyte maturation result subsequent to imbalance of any of these intraovarian factors [29]. Studies carried out in 2016 demonstrated that observation of BMP2, BMP4, BMP7, BMP15, GDF8 along with GDF9 was seen in the follicular fluid [21]. Of these BMP s from follicular fluid the outcomes from the clinical data pointed that BMP2 as well as BMP15 might get utilized as probably good pointers of oocytes fertilization along with/ or oocytes maturation. along with Prior work had revealed the expression of mRNA as well as protein of BMP receptors in different follicular partitions of preantral along with antral follicles of the human ovary $[26,27]$. Of these receptors maximum expression is of BMPR2, type II receptor [27]. In contrast to type I receptors, ALK1, ALK2, as well as ALK7the expression of4 type I receptors like ALK3, ALK4, ALK5 as well as ALK6 was in modest amounts in human preantral follicles [27]. In a study in 2016 it was illustrated that the impairment of ALK6 in human GCs is correlated with reduction in the ovarian reserve as well as age associated reduction in fertility [30].

\section{Primordial Germ Cell Generation as well as BMPs}

Primordial Germ Cells (PGCs), that are the precursors of sperm along with egg in the adult, represent a group of germline stem cells, which get generated just at the time of early embryonic stage [6]. Migration of these Stem Cells towards the primitive gonadal fold in addition to mitotic proliferation for escalation of cell amounts whose differentiation takes place following that into Primordial follicles (oocytes correlate with the somatic cells [6]. BMP sub family members of have a key role in controlling ovarian generation as well as function [6]. Nevertheless, the generational events were differentially controlled in rodents along with humans with regards to the spatiotemporal organization [31]. In case of mouse embryos, the extraembryonic ectoderm obtained BMP 4 as well as BMP 8in cooperation stimulated the generation of PGCs [6] In human fetal ovaries, BMP 4 as well as BMP signaling manipulated post migratory PGC amounts by facilitation of apoptosis [31]. at the time of oogenesis in humans transient GDF 9 liberations by oocytes prior to follicle generation, that is correlated with activin $\beta$ A signaling from somatic cells for establishing selective germ cell survival [6].

\section{Intraovarian cell-cell connection as well as BMPs}

The ovarian follicle is the dominant functional unit in the context of female reproductive system. A synchronized crosstalk amongst, this biological compartment amongst the oocytes as well as follicular cells (cumulus cells/GCs as well as theca/stroma cells) is dependent robustly on functional gap junctions. These connexin coupled cell junctions directly modulates cell- cell connection by letting the small molecules (ions, metabolites, nutrition as well as small signaling molecules) amongst, two cells existent next to one another (rev in 6). In case of humans the growing follicles, connexin 43 ( $\mathrm{C} \times 43$ ) primarily aids in the generation of gap junctions amongst cumulus cells/GCs, while the gap junctions which aid in the communication of the oocytes with the surrounding cumulus cells posses 37s connexin 37 ( $\mathrm{C} \times 37$ ) as their major constituents (rev in 6). in the knockout mice studies illustrated those mice with absence of $\mathrm{Cx} 43$ possessed a phenotype with reduction in germ cell amounts in the fetal gonad, retardation of growth of follicles besides failure of fertilization (rev in 6). Additionally, ablating Cx37 resulted in depletion of intercellular coupling amongst oocytes as well as cumulus cells, impairment of follicle generation at the antral stage, oocytes that are incompetent in addition to impairment of ovulation (rev in 6).

Cx43 expression is massive in the GCs all through the follicular stages, with there being need of their expression in the context of GC proliferation (rev in ref 6). The C x 43 expression in a cyclic fash- 
An Update on the Role of Bone Morphogenetic Protein and Growth Differentiation Factor on Follicular Generation in Human Menstrual Cycle with Clinical Impact on ART Success and PCOS Pathogenesis/Therapy-a Systematic Review

ion in the GCs gets generationally along with hormonally controlled by gonadotrophins, (FSH as well as $\mathrm{LH}$ ) besides steroid hormones (estrogen - androgens, progesterone) in numerous species inclusive of humans (rev in 6). The studies by Chang HM., et al. [32], have illustrated that 3 intraovarian BMPs (BMP4, BMP7 as well as BMP15) in addition to TGF- $\beta$ might work in an autocrine/paracrine fashion in the context of modulation of $\mathrm{Cx} 43$ expression besides intercellular connection in human GCs (Figure2) [31]. In particular BMP modulated signaling results in repression of $\mathrm{Cx} 43$ expression, while TGF- $\beta 1$ causes escalation of $\mathrm{Cx} 43$ expression, that pointed that there is existence of competing controlling parts of these autocrine/paracrine factors. The functional differences amongst the growth factors that represent TGF-beta superfamily of the differential signaling molecules (TGF- $\beta$ as well as BMPs) might impact numerous cellular functions.

\section{Crosstalk amongst Oocytes-somatic cells}

In case of mammalian ovary, oocytes do not simply stay within the follicles, besides being in receipt of generational signals from the cumulus/granulosa cells that are surrounding them, however they have active participation in regulation along with modulation of follicular generation in addition to ovulation. In the follicular milieu, Oocytes as well as the -somatic cells that confer support in synchronization regulate the generation along with maturation of the follicle in addition to acquiring a meiotically competent oocyte (rev in 6). The outcomes derived from animal studies pointed that oocyte might cause facilitation of follicular generation, besides, cell differentiation by liberation of certain growth factors which work in a paracrine fashion for impacting the adjacent cumulus/ granulosa cells (GCs). In turn these paracrine action result in modulation of oocytes generation along with maturation [33]. Studies that were conducted in sheep as well as mice have illustrated that the experimental destruction (remove, absence or destroy) of the oocytes caused dysregulated folliculogenesis, which pointed that there is existent, a key part of the oocytes factors or oocytes induction of paracrine signaling in follicular generation (rev in 6). A in vivo study documented, that ovectomy possesses the capacity of spontaneous conversion of graafian follicle into the corresponding corpus luteum (rev in 6). It has been illustrated in in vitro studies, that oocyte liberated factors possess the potentiality of facilitation of DNA generation as well as a cell proliferation in the surrounding cumulus/mural GCs (rev in 6). Additionally, these oocytes obtained robust mitogens might further result in escalation of the actions of various GCs controllers (like FSH, insulin like growth factor1 as well as androgens) or cell growth actions (rev in 6).

the capacity of robust modulation of cumulus/GCs differentiation in different species, inclusive of humans [32epp]. Specifically, modulation of FSH stimulated estrogen -progesterone generation by GCs (rev in 6), while they result in repression of the expression of LH receptor (LHR) whose induction is caused by FSH in GCs (rev in 6). Besides gonadotrophins liberated by the pituitary gland, oocytes obtained BMP15 as well as GDF9 further take part in manipulation of some of the target genes that are associated with ovulation along with luteinization (rev in 6). As far as human GCs are concerned, oocytes obtained BMP15 results in reduction of progesterone generation by down regulation of the expression of StAR [34]. In total these outcomes corroborate, an earlier posit that the oocytes possess the capacity of hampering follicular luteinization (Figure2) (rev in 6).

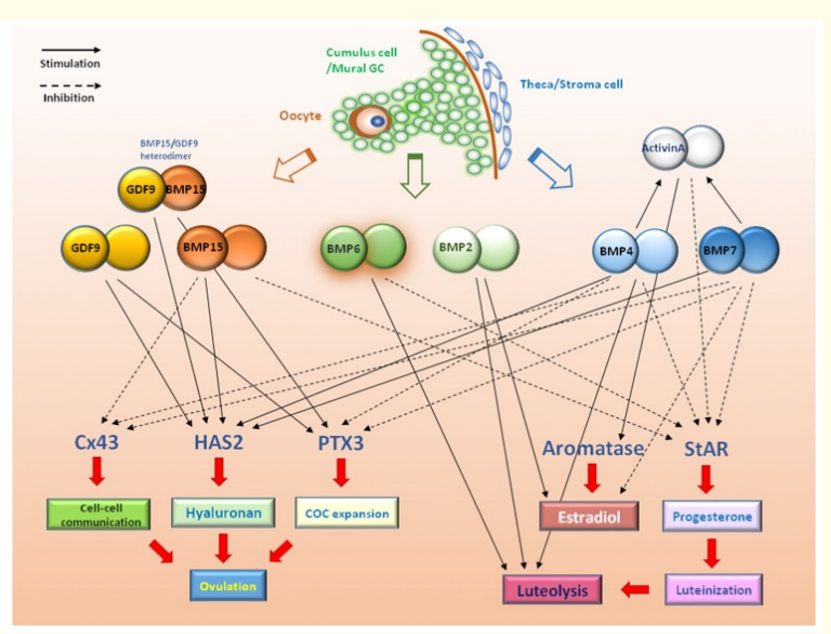

Figure 2: Courtesy ref no-6- Schematic diagram summarizing functional roles of BMPs and GDF9 in the human ovary. The potential physiological roles of intra-ovarian BMPs in regulating human ovarian functions, including steroidogenesis, activin

production, cumulus-oophorus complex formation and expansion, cell-cell communication, ovulation and luteolysis are shown. BMP, bone morphogenetic protein; COC, cumulus-oophorus complex; Cx43, connexin 43; GC, granulosa cell; HAS2,

hyaluronan synthase type 2; PTX3, pentraxin 3; StAR, steroidogenic acute regulatory protein. 
An Update on the Role of Bone Morphogenetic Protein and Growth Differentiation Factor on Follicular Generation in Human Menstrual Cycle with Clinical Impact on ART Success and PCOS Pathogenesis/Therapy-a Systematic Review

Results that have accumulated from the cumulus cell transcriptome, illustrated that bidirectional crosstalk amongst, the human oocyte as well as cumulus cells is, necessary for the generation, of a competent oocyte (rev in 6). In this context, the oocyte works in the form of central controller of the adjacent follicular cell function by liberation of different growth factors as well as cytokines for the modulation of cell proliferation, cell differentiation, apoptosis as well as luteinization (rev in 6).

During ovulation cumulus- expansion is a complex event, needed for the ideal expulsion of the cumulus -cell mass from the follicle (rev in 6). The cumulus- expansion event, besides oocyte maturation are significantly based on the crosstalk of 2 signals whose induction occurs by the epidermal growth factors (EGF) like peptides (whose triggering occurs secondary to LH or HCG) in addition to oocyte- obtained paracrine factor [35]. In particular the oocyteobtained paracrine factor causes capacitation of the reaction of cumulus cells to $3 \mathrm{EGF}$ ) like peptides, that result in induction of expression of numerous target genes [HAS2, Tumor necrosis factor alpha-induced protein 6 (TNFAIP6), pentraxin 3 (PTX3), prostaglandin endoperoxidase synthase 2 (PTGS2) that are associated with extracellular matrix [ECM] generation (rev in 6). Actually, the in vitro studies have demonstrated that oocyte factors GDF9, with lesser degree BMP4 and BMP7 cause induction of HAS2 expression in addition to hyaluan along with prostaglandin E2 generation that is necessary for normal, ovulation [16]. The insight obtained from cumulus -oocyte crosstalk will aid clinicians fashioning of ideal procedures in particular the protocol with regards to IVM.

\section{Premature luteinization avoidance by BMPs}

The LH surge in humans' results in stimulation of numerous intrafollicular actions along with triggering ovulation (rev in 6). During ovulation numerous steps of morphological alterations as well as tissue remodeling those results in rupture of the ovarian follicle along with generate a corpus luteum, an endocrine structure which liberate progesterone (rev in 6). This progesterone liberation, besides targeting results in the preparation of the reproductive tract for the start of fertilization as well as sustenance of early pregnancy (rev in 6). Premature luteinization implies escalation of progesterone amounts on or the day prior to HCG delivery in case of patients who are recipients of controlled ovarian stimulation (rev in 6). This premature enhancement of progesterone can switch the im- plantation window (harmonization amongst, embryonic generation as well as endometrial receptivity) that might hinder embryo implantation addition to result in reduction of pregnancy rates. At the time of antral follicle stage, one of the maximum significant physiological functions is the avoidance of premature luteinization that causes sustenance of follicular growth besides somatic cells proliferation (rev in 6).

The proof obtained from animal studies have illustrated that the control of steroid hormones BMP's is species as well as stage particular in preantral as well as antral follicle stages. In case of human's certain outcomes that have not been reproducible got published in the context of the controlling actions of BMP's on ovarian steroid hormones generation. In case of a human granulosa tumor cell line (KGN), BMP4 and BMP7 cause repression of forskolin or cAMP stimulated progesterone generation, without having impact on forskolin or cAMP stimulated estradiol (E2) generation (rev in 6). Conversely, utilization of GCs in a cohort study from normal, healthy women along with, PCOS women demonstrated that BMP7, however not BMP4 resulted in repression of E2 generation as well as BMP4 and BMP7 both possessed no action, on FSH stimulated E2 generation in normal women [26khalaf]. Where utilization of primary GCs was done in studies that were obtained from IVF patients illustrated that BMP2, BMP4, as well as BMP6 resulted in repression of StAR expression, while BMP2 caused induction of aromatase expression [28]. In agreement with certain prior studies, later outcomes of Chang., et al. studies illustrated that BMP4, BMP7 as well as BMP15all resulted in reduction of progesterone generation through down regulation of ALK5 mediated StAR expression in immortalized (GCs) (SVOG), however not in KGN cells [36,37]. Furthermore, BMP4 as well as BMP7 escalated the generation of bioactive activin A through the up regulation of in addition to proteolytic processing of inhibin $\beta A$ subunit in human immortalized GCs [22]. Activin A represents another robust agent for hampering in view of its capacity of reduction of basal along with FSH/LH stimulated progesterone generation in hGL cells [34]. GDF, that is an intricate associate of BMP 15, possesses an opposed part in the controlling of human steroid hormones generation. With regards to human GCs as well as theca cells, GDF9causes repression of 8 bromo- cAMP induction of StAR expression along with progesterone generation without impacting the StAR protein amounts or progesterone generation (rev in 6). 
An Update on the Role of Bone Morphogenetic Protein and Growth Differentiation Factor on Follicular Generation in Human Menstrual Cycle with Clinical Impact on ART Success and PCOS Pathogenesis/Therapy-a Systematic Review

In toto all studies in human follicle cells point that liberation of BMPs from oocytes occurs. GCs/theca/stroma cells hamper StAR expression that in return results in reduction of progesterone generation. Conversely, oocyte obtained GDF9 cross talks with primary gonadotrophins for further decreasing progesterone generation (Figure2). Subsequent to ovulation, the massive reduction in GDF9 as well as BMP 15 amounts in the corpus luteum results in escalation of StAR expression as well as subsequent escalation of progesterone generation.

\section{Modulation of COC generation as well as expansion}

At the time of antral follicle stage (that has the properties of a fluid filled antrum), the initial GCs which are surrounding the oocyte get differentiated into 2 functionally in addition to anatomically different sub lineages; the cumulus cells closely crosstalk with the oocyte to generate a distinct large structure known as $\mathrm{COC}$, ii) the mural GCs line the follicular wall with the generation of layers besides being close to the neighbouring basement membrane, as well as theca/stroma cells (rev in 6). At the time of periovulatory stage, these 2 kinds of GCs display markedly disparate properties. Usually, cumulus cells are rich in transcripts implicated in cell proliferation as well as, metabolism while transcripts mural GCs are rich in transcripts implicated in cell differentiation along with signaling [38]. As compared to mural GCs, the cumulus cells exhibit a greater cell proliferation rate, greater expression of $\mathrm{AMH}$, lesser steroidogenic capacity, lesser expression of LHR amounts, besides possessing the capacity of liberation of hyaluronic acid for COC expansion (cumulus expansion) (rev in 6). The cumulus cells correlate with the ovary in addition to the extended viscoelastic ECM to generate a solitary structure, the hyaluronan-enriched COC matrix (rev in 6). Hyaluronan acts as a structural backbone of this matrix, whose stabilization further gets attained by a complicated network of binding protein, that are inclusive of versican, TNF stimulated gene 6 protein (TSG), inter - $\alpha$-trypsin inhibitor as well as PTX [39].

During the midcycle LH surge, the event of ovulation gets originated by cell-cell connection, with the resumption of oocyte meiotic maturation, that causes tissue remodeling in addition to induction of the expansion of the COC (rev in 6). The cumulus cells expansion, that possesses the properties of induction of morphological alterations, is implicated in the proliferation along with dispersing of cumulus cells gets started on getting regulated by different endocrine, paracrine in addition to oocyte obtained factors. [34].
The need of the oocyte or its conditioned medium was illustrated by in vitro studies in the context of response of cumulus cells to EGF, FSH, or cAMP in the generation of hyaluronan [38]. in cumulus cells, the crucial enzymes implicated in the polymerization as well as elongation of the hyaluronan chains for their localization into the intercellular space is a transmembrane protein HAS2 (rev in 6). Actually, the human BMP 15 homodimers as well as GDF9 possess the capacity of up regulation of the cumulus expansion associated genes (Has2, Ptx3 as well as Ptgs2) in mouse GCs for facilitation of cumulus expansion in vitro [11].

With regards to the structure of the hyaluronan dependent ECM, PTX3 possesses key part in the event of assembly. This network that is stable besides being sustainable hyaluronan network, PTX3 works in the form of an accumulating reagent for getting attached to a molecule, TSG6, that is bound further to the separate hyaluronan strand (rev in 6). For emphasizing the necessary part of PTX3 as well as cumulus expansion in the event of oocyte maturation as well as subsequent ovulation, the effectiveness of oocyte transportation via the oviducts along with invitro fertilization (IVF) the knockout mouse model has been utilized (rev in 6). Mice with absence of Ptx3 in human reveal subfertility, structural aberrations of COC along with failure of IVF (rev in 6). Chang HM., et al. [36], study demonstrated that BMP4 as well as BMP7 resulted in downregulation of the expression along with protein generation of pentraxin 3 in human GCs, that pointed that BMPs might take part in the ECM generation along with tissue remodeling. Totally one can posit that oocyte liberated factors (mainly BMP 15 as well as GDF 9) result in sustenance of the cumulus cells phenotype by facilitation of cumulus expansion, while the human preantral follicle function is where that are while the theca/stroma while the theca/stroma obtained BMPs (mainly BMP4 and BMP7) cause decomposition of the structure of ECM in the adjacent mural GCs for promotion of the separation of the COC along with mural GCs in the human ovary.

\section{BMPs as well as luteal function}

If pregnancy does not result, the corpus luteum starts regressing during the end of the luteal phase, a breakdown event known as luteolysis (rev in 6). Luteolysis, is a complex event, implicating deletion of the structural as well as functional intactness of the corpus luteum that is associated with a reduction in progesterone generation (rev in 6). 
An Update on the Role of Bone Morphogenetic Protein and Growth Differentiation Factor on Follicular Generation in Human Menstrual Cycle with Clinical Impact on ART Success and PCOS Pathogenesis/Therapy-a Systematic Review

Studies with utilization of human ovarian tissues illustrated that the expression amounts of BMP2, BMP4 as well as BMP6 are escalated at the time of luteal regression, besides being differentially controlled by HCG, that points that BMPs might be implicated in the event of luteolysis [28]. (Figure2).

\section{BMPs part in female reproductive pathology}

Any abnormalities in the intraovarian BMPs or BMPs signaling might negatively impact oocyte-somatic cells crosstalk, steroidogenesis, GCs proliferation, oocyte maturation, cumulus expression, ovulation, embryonic quality along with luteal function that results in infertility in addition to reproductive pathology. On knocking out of Bmp4 or deletion of Alk2 in mouse embryos causes absence of generation of PGC (rev in 6). On genetic ablation of the Bmp6 gene in female mice causes a reduction of the amounts of ovulated eggs along with reduction of the litter size (rev in 6). Oocyte obtained BMP 15 as well as GDF 9 are the distinct BMP/GDF correlation with different ovarian functions along with ovulation rate [12]. Aberrations in expression of these 2 factors might be associated with female infertility (rev in 6). The outcomes from clinical data pointed that the utilization of BMP 15 amounts in follicular fluid might act as a pointer of oocyte quality in addition to following fertilization capacity (rev in 6). Additionally, 2 BMP receptors have been demonstrated to be implicated in implantation along with maternal -fetal crosstalk at the time of human pregnancy. Impairment of the BMPR2 modulated signaling in the uterine decidua results in placental abruption, fetal death as well as female infertility (rev in 6). At the time of mouse pregnancy, mice possessing (Alk2 cKO mice) illustrated delayed invasion into the uterine stromal failure of uterine decidualization as well as infertility. Similarly, ALK2 ablation with the utilization of siRNA that was targeted to ALK2 in human uterine stromal cells might change uterine decidualization as well as embryon implantation, resulting in infertility (rev in 6).

\section{Polycystic ovary syndrome}

With the utilization of Immunohistochemical staining, the expression amounts of BMP 15 as well as GDF 9 proteins in the oocyte along with GCs of follicle in polycystic ovary syndrome (PCOS) ovaries are decreased at the time of early follicular phase [40]. In contrast to the control group, the expression amounts of GDF 9 proteins in the oocyte possessed a tendency to be greater in PCOS women, that might have been secondary to PCOS follicular impair- ment (rev in 6). Nevertheless, the expression amount of GDF 9 proteins in cumulus cells are lesser in PCOS women that might result in premature luteinization, bad oocyte competence along with luteal impairment, causing greater miscarriages in patients of PCOS (rev in 6). These outcomes obtained yield 2 important intraovarian growth factor in the controlling of oocyte maturation in the follicular milieu along with would aid in fashioning of a probable strategy of enhancement of IVM protocols for oocytes from PCOS women [29]. Maximum serum BMP protein amounts are either absent or existent at the lower amounts of detection in PCOS women, thus yield minimum knowledge in the context of this disease. GWAS (genome wide association studies) data point that the implication of BMP 15or GDF 9 genetic differences in PCOS etiology continue to be debatable [12]. More recently, in a mouse model of PCOS, Asghari R., et al. s observation was that there was changed gene expression in preantral follicles of TGFB1, GDF9and BMPR2 genes that got induced byes radio valerate [41].

\section{Primary ovarian insufficiency}

Various BMP 15 gene mutations have been documented in Primary ovarian insufficiency (POI) patients with manifestation as primary or secondary amenorrhea (rev in 6). The first missense mutation in the BMP 15 gene was isolated in 2 Italian sisters with familial ovarian dysgenesis as well as primary amenorrhea (rev in 6). in contrast to the normal human BMP 15 protein, a recombinant BMP 15 generated from this mutant gene could not cause stimulation of growth action of GCs, besides inability of hampering of progesterone generation in primary human GCs (rev in 6). Akin to BMP 15, various cohort studies implicating several POI have been done, for screening of GDF 9 mutations [42]. Nevertheless, different missense mutations that encode for various GDF 9 mutant proteins might reveal opposing outcomes in functional studies. Certain variants significantly cause reduction in mature GDF 9 protein generation, along with cause reduction in the capacity of GDF 9 to stimulate GCs proliferation (rev in 6). Conversely, other variants might encode a mutant GDF 9 proteins that significantly causes escalation of GCs proliferation [42].

\section{Endometriosis}

The definitions of endometriosis is the existence of endometrial tissue beyond the uterine cavity, that impacts $10 \%$ of the reproductive aged women. Despite, numerous posits, the exact pathogenesis 
An Update on the Role of Bone Morphogenetic Protein and Growth Differentiation Factor on Follicular Generation in Human Menstrual Cycle with Clinical Impact on ART Success and PCOS Pathogenesis/Therapy-a Systematic Review

as well as molecular modes of this common disease is not certain. Immunohistochemical staining of these endometrial lesions received from 85 patients demonstrated that robust expression of BMP6 in both epithelial as well as stroma cells occurred (rev in 6). A genome wide profiling evaluation that contrasted the ectopic along with eutopic endometrial tissues illustrated that BMP4 along with its antagonist gremlin (GREM1), are impaired in endometriosis. Furthermore, studies evaluating the functional part of BMPs in the generation of endometriosis would be of good use.

Innovative part of GDF8 in the human ovary

To start with isolated in the musculoskeletal system GDF8 belongs to the TGF- $\beta$ superfamily. GDF8 as well as BMP11 (GDF11) represent 2 intricately associated growth factors, sharing akin structure along with function. Furthermore, given the name of myostatin, GDF8 is a robust hampering agent of skeletal muscles growth along with generation. Mice possessing a targeted mutation of the Gdf8 gene possess an escalation equivalent to $25-30 \%$ of muscles mass in view of hypertrophy in addition to hyperplasia (rev in 6). GDF8 gene mutations occurring naturally resulting in escalation of muscles mass have been documented in different species, inclusive of cattle, sheep, dogs, as well as humans. During the last decade, escalation of attention in the evaluation of the functional part of GDF8 beyond the musculoskeletal system have been documented. Noticeably the expression along with probable functions of GDF8 have been conducted more recently in numerous reproductive organs inclusive of, uterus as well as placenta (rev in 6).

GDF8 as well as ovarian steroidogenesis

In case of chicken embryos there is abundant expression in various tissues inclusive of testis as well as ovary. In case of bovine ovaries, microarray displayed GDF8 expression in the GCs of various sizes of antral follicles (rev in 6). Unpublished data from Chang., et al. laboratory documented that GDF8 as well as its functional receptor (ACVR2A, ACVR2B as well as ALK5) expression occurs in GCs of growing follicles in the human ovary [Unpublished]. Furthermore, the mature GDF8 protein can get estimated in the follicular fluid garnered from IVF patients (rev in 6). Additionally, an Innovative part of GDF8 in the controlling of human GCs steroidogenesis. In particular GDF8 controls steroid generation by escalation of aromatase/estradiol (E2) generation, whereas causes reduction of StAR/progesterone generation in primary hGL cells
[43]. evaluation of the results from clinical samples corroborates these observations, in view of negative association amongst GDF8 amounts along with progesterone amounts in the serum as well as follicular fluid [18]. Furthermore, GDF8 might cause escalation of FSH- stimulated aromatase/estradiol (E2) generation, while GDF8 might be resulting in repression of LH stimulated StAR/progesterone generation in hGL cell (rev in 6). With regards to this GDF8 influences a controlling function by modulation of GCs responsiveness to gonadotrophins. Noticeably, GDF8 escalation of GCs responsiveness to FSH by up regulation of FSH receptor (FSH R) expression, whereas causes repression of GCs responsiveness to LH by downregulation of LHR expression [43]. In agreement with these outcomes the prior outcomes of Change., et al. illustrated that activins (activin A, B, AB) might work in akin fashion for modulation of steroidogenesis in same cell model [44]. The downstream signaling pathway might describe the akin cellular actions as both GDF8 as well as activins work through SMAD 2/3- SMAD 4 modulated target gene regulation in human GCs $[8,44]$.

\section{GDF8 as well as GCs proliferation}

At the time of follicular generation, GCs proliferation in addition to terminal differentiation of GCs represent crucial events that are necessary for oocyte maturation, subsequent ovulation as well as luteinization (rev in 6). During the periovulatory stage, different controlling endocrine as well as paracrine factors with great precision cause the modulation of functional shift of GCs from a significantly proliferative status to one of a non-proliferative, terminally differentiated status (rev in 6). Further than its part in control of steroid generation, an in vitro study illustrated that GDF8 results in repression of human GCs proliferation [24]. This negative controlling function modulation occurs by a separate growth factor, namely Connective tissue growth factor (CTGF), pointing that the GDF8 along with CTGF might be implicated in the regulation of markedly proliferative in addition to non-proliferative actions at the time of human follicular generation [24].

\section{GDF8 as well as extracellular matrix generation}

Lysyl oxidase (LOX) represent crucial enzyme for the ultimate assembling along with stabilization of the extracellular matrix [ECM], that is necessary for follicle along with oocyte maturation (rev in 6). In case of rat ovary LOX expression amounts are positively associated with quality of oocytes (rev in 6). In numerous organs 
CTGF is the key modulator of ECM- associated tissue remodeling (rev in 6). CTGF along with LOX expression takes place in human GCs, with CTGF modulation of the GDF8 stimulated escalation of LOX) action as well as LOX protein expression [23]. Intriguingly, the GDF8 stimulated up regulation of CTGF expression in human GCs takes place via the ALK5 modulated SMAD 2/3- SMAD 4 signaling pathway [23]. This observation is not in agreement with an earlier study that illustrated GDF8 signaling was based on ALK4 however not ALK5 in mouse C2C12 myoblasts, that pointed that type I receptor modulated downstream signaling is cell kind particular (rev in 6). In particular GDF8 works via ALK4 in myoblasts cells whereas ALK5 modulates the GDF8 stimulated cellular action in non-myogenic cells.

\section{GDF8 as well as cumulus expansion}

That the taking place of COC besides the degree of cumulus expansion have been associated with the competence of oocytes along with probably of use in the form of pointers in the context of oocyte selection in the IVF program. The combination of outcomes of in vitro studies as well as the clinical results further documented a positive association amongst PTX3 expression amounts in cumulus cells in addition to the oocytes quality which corresponded with them as well as subsequent fertilization (rev in 6). In agreement with the actions of BMP4, along with BMP7, GDF8 might be implicated in modulation of cumulus expansion by downregulation of the vital associated protein, PTX3 in human GCs [21].

Collectively GDF8 expression takes place in human GCs, with its mature proteins can get estimated in follicular fluid. Stepwise functional studies have illustrated the part of GDF8 in control of steroidogenesis, responsiveness to gonadotrophins, LOX expression, LOX action in addition to PTX3 expression in human GCs (Figure3). These observations point that this individual TGF-beta superfamily members might possess key part in the ultimate differentiation event in the growing follicles, that is most probably by working for maturation stimulation in addition to hampering of luteinization.

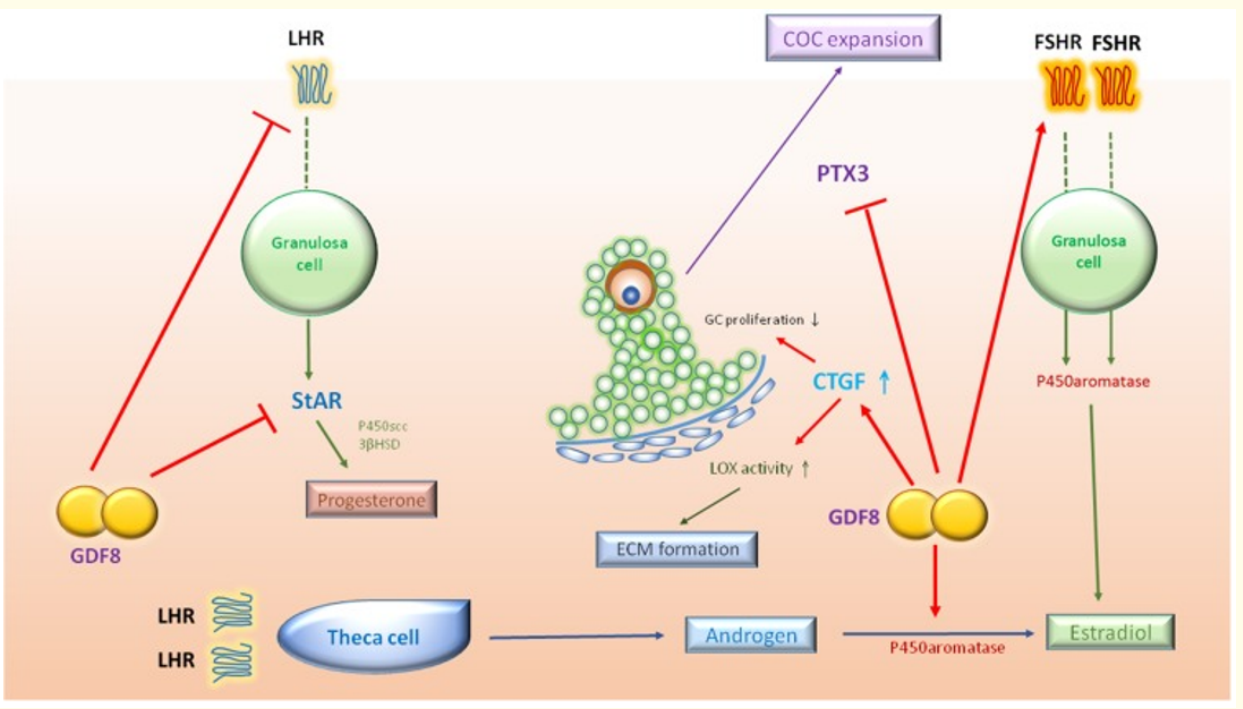

Figure 3: Courtesy ref no-6- Schematic diagram summarizing potential roles of GDF8 in a human growing

follicle. In this follicular microenvironment, the locally produced GDF8 may promote aromatase/estradiol and FSHR

expression, suppress StAR/progesterone and LHR expression and down-regulate PTX3 expression. In addition, GDF8 induces the

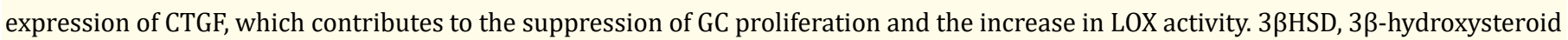
dehydrogenase; CTGF, connective tissue growth factor; ECM, extracellular matrix; LOX, lysyl oxidase; P450scc, P450 side-chain cleavage enzyme; PTX3, pentraxin 3; FSHR, FSH receptor; LHR, LH receptor. 
An Update on the Role of Bone Morphogenetic Protein and Growth Differentiation Factor on Follicular Generation in Human Menstrual Cycle with Clinical Impact on ART Success and PCOS Pathogenesis/Therapy-a Systematic Review

Therapeutic potential along with future perspectives

A greater exhaustive insight in the context of physiological functions of BMPs/GDF the human ovary would yield important understanding along with provide novel approaches for development of new strategies, with regards to fertility control, with the objective for generation of alternative methods of contraception, for diagnosis along with treatment of human fertility, or for generation of protocols that are safer in addition to reliable for the induction or controlled ovarian stimulation (COS) assisted reproductive technology (ART). The implication of BMPs/GDF signaling pathways in a wide array of generational as well as pathophysiological events of reproductive biology one can target these signaling pathway in the form of a probable therapeutic strategies for infertility therapy. Existently, various recombinant human BMPs/GDF along with their hampering agents or antagonist have gone via clinical trials or have got the approval of US FDA in human skeletal as well as muscular systems. In line with that the group of Chang HM., et al. have illustrated novel insight into the molecular modes by which BMP2 modulation of the cellular actions that is stimulated by GDF via upregulation of expression in human granulose-lutein cells of their antagonist induction of cell signaling gremlin 2 (GREM2). [45]. Moreover, the latest generation of gene therapies as well as BMP ligand trapping strategies, are assuming interest. The whole knowledge acquisition points distinctly the availability of potential of new medicines that work on the BMPs/GDF signaling pathways for the therapy of numerous human diseases, that is inclusive of, female reproductive diseases. Nevertheless, further human clinical trials are required for evaluation of effectiveness, safety along with route of delivery of these Pharmacological applications.

The results of Bai., et al. [46] in a study in the context of exploration of poor ovarian reserve- associated risk factors for getting insight into the potential role of Growth Differentiation factor 8 in the anticipation of ovarian response in IVF-ET patient, illustrated that besides implication of different factors having significant impact poor ovarian response (POR), including aging, obesity, endometriosis, surgery history, and IVF treatment, there was a reduction in the expression amounts of ACVR2A in POR patients. Additionally, clinical association evaluation outcomes illustrated a negative association with GDF 8 amounts with LH as well as estradiol amounts along with, antral follicles counts. Thus, there study gave an innovative part of GDF 8amounts in ovarian response in $\mathrm{COH}$ event. Gong., et al. [47], observed that the expression level of GDF9 and BMP15 genes in the FF and GCs varied significantly among the three groups $(\mathrm{P}<0.05)$, from ages $(<35$ year, $35-40$ year $(>40$ year, demonstrating a trend of decline along with age. The ratio of GDF9/BMP15 mRNA levels were akin among the three groups (P $>0.05)$. The relative amounts of GDF9 along with BMP15 proteins in GCs associated with the relative mRNA levels in GCs and protein amounts in FF $(\mathrm{P}<0.05)$. Thus they concluded that for poor ovarian responders, specifically those over 40 , the expression of GDF9 in addition to BMP15 was reduced in addition to escalated age besides being associated with poorer oocyte quality along with IVF results, while the ratio of GDF9/BMP15 mRNA amounts continued to be relatively constant. Furthermore, Turathum B., et al. [48] reviewed the role of cumulus cells in oocyte, growth, maturation, followed by ovulation in addition to subsequent fertilization with elaboration of roles of BMPs/GDF system. Since cumulus cells are implicated in the realization of oocyte meiotic maturation ovulation in addition to subsequent fertilization insight in functions of cumulus cells might aid in anticipation of oocyte quality besides embryonic generational competence in addition to pregnancy outcomes in the field of reproductive endocrinology as well as ART. Magro-Lopez E, Munoz-Fernandes further reviewed the part of BMPs signaling in the context of female reproductive system generation in addition to function with their Clinical relevance in treatment of female reproductive diseases[49]. Furthermore, Bai., et al. [50], demonstrated, that abnormalities of glucose metabolism occurred in granulosa cells of PCOS patients, secondary to up regulation of SERPINE expression due to escalation of GDF8 [50].

\section{Conclusions}

Over the last decade, investigators have been studying the locally generated growth factors in addition to intrafollicular signaling amongst, the ovary as well as follicle cells has evoked marked attention. The data accrued point that intraovarian BMPs/GDF system is significant in regulation of female reproduction that is inclusive of, PGC generation, cell-cell contact, steroidogenesis, COC generation as well as expansion, oocyte maturation, subsequent ovulation along with luteolysis. Hence the association amongst oocyte along with its supporting cells (GCs as well as theca/stroma cells) need to be considered in the form of a synchronous association. At the time of follicular generation, all of these cell kinds have a necessary part, however unique part, in control of ovarian 
functions for the generation of an oocyte that is competent for the embryo generation. An exhaustive insight of the expression, effects away molecular modes of the BMPs/GDF system in the human ovary is key for the generation of clinical strategies in the context of diagnosis along with treatment of women with presentation of infertility along with ovulatory impairment.

\section{Bibliography}

1. Knight PG and Glister C. "TGF-beta superfamily members and ovarian follicle development". Reproduction 132 (2006): 191206.

2. Wagner DO., et al. "BMPs: from Bone-to-Bone morphogenetic proteins". Science Signaling 3 (2010): mr1.

3. Rossi RO., et al. "The Bone morphogenetic protein system and the regulation of ovarian follicle development in mammals". Zygote 24 (2016): 1-17.

4. Qin Y., et al. "Genetics of primary ovarian insufficiency: new developments and opportunities". Human Reproduction Update 21 (2015): 787-808.

5. Rider CC and Mulloy B. "Bone morphogenetic protein and Growth Differentiation factor and cytokine families and their protein antagonists". Biochemical Journal 429 (2010): 1-12.

6. Chang HM., et al. "Oocyte somatic cell interactions in the human ovary-a novel role of Bone morphogenetic proteins and Growth Differentiation factors". Human Reproduction Update 23.1 (2017): 1-1-8.

7. Veitia RA and Caburet S. "Extensive sequence turnover of the Signal members of GDF/ BMP family: exploring their evolutionary landscape". Biology Direct 4 (2009): 22.

8. McIntosh CJ., et al. "The proregion of mouse BMP15 regulates the cooperative interactions of BMP15 and GDF9". Biology of Reproduction 79 (2008): 889-896.

9. Seidah NG and Prat A. "The biology and the therapeutic targeting of proproteins convertases". Nature Reviews Drug Discovery 11 (2012): 367-383.

10. Harrison CA., et al. "Prodomains regulate the synthesis, extracellular localization and activity of TGF-beta superfamily ligands". Growth Factors 29 (2011): 174-186.

11. Peng J., et al. "Growth Differentiation factor9, Bone morphogenetic protein 15 heterodimers are potent regulators of ovarian function". Proceedings of the National Academy of Sciences of the United States of America 110 (2013): E776-785.
12. Persani L., et al. "The fundamental role of Bone morphogenetic protein 15 in ovarian function and its involvement in female fertility disorders". Human Reproduction Update 20 (2014): 869-883.

13. Miyagi M., et al. "BMP2, BMP4, noggin, BMPR1A, BMPR1B, and BMPRII are differentially expressed in the adult rat spinal cord". Neuroscience 203 (2012): 12-26.

14. Mueller TD and Nickel J. "Promiscuity and specificity in BMP receptor activation”. FEBS Letters 586 (2012): 1846-1859.

15. Holdzhausen A., et al. "Novel Bone morphogenetic protein signaling through Smad 2 and Smad 3 to regulate cancer progression and development". The FASEB Journal 28 (2014): 12481267.

16. Zhang H., et al. "Differential activation of non-canonical signaling of SMAD2/SMAD3 by Bone morphogenetic proteins causes disproportionate hyaluronan production in immortalized granulosa cells". Molecular and Cellular Endocrinology 428 (2016): 17-27.

17. Yamaguchi K., et al. "Identification of the MAPKKK family as a potential mediator of TGF-beta signal Transduction". Science 270 (1995): 2008-2011.

18. Fang L., et al. "Growth Differentiation factor 8 decreases StAR expression through ALK5 mediated Smad 3 and ERK1/2 signaling pathways in luteinized granulosa cells". Endocrinology 156 (2015): 4284-4294.

19. Bayasula Iwase A., et al. "Establishment of a human non luteinized granulosa cell lines that transitions from the gonadotrophins independent to the gonadotrophins dependent status". Endocrinology 153 (2012): 42851-42860.

20. Fang L., et al. "TGF-beta 1 increases lysyl oxidase expression by down regulating MIR29A in human granulose-lutei n cells". Reproduction 152 (2016): 205-213.

21. Chang HM., et al. "Recombinant BMP4 and BMP7 downregulate pentraxin 3 in human granulosa cells". The Journal of Clinical Endocrinology and Metabolism 100 (2015a): E365-374.

22. Chang HM., et al. "Recombinant BMP4 and BMP7 increase activin A production by up regulating inhibin beta subunit and furin expression in human granulose-lutein cells". The Journal of Clinical Endocrinology and Metabolism 100 (2015c): E375386. 
23. Chang HM., et al. "Activin A induced increase in LOX activity in human granulose-lutein cells is mediated by CTGF". Reproduction 152 (2016a): 293-301.

24. Chang HM., et al. "Connective tissue growth factor mediates Growth Differentiation factor 8- induced increase of lysyl oxidase activity in human granulose-lutein cells". Molecular and Cellular Endocrinology 434 (2016c): 186-198.

25. Li Y., et al. "Increased GDF 9 and BMP 15 mRNA levels in cumulus granulosa cells correlate with oocyte maturation, fertilization and embryo quality in humans". Reproductive Biology and Endocrinology 12 (2014): 81.

26. Khalaf M., et al. "BMP system expression in GCs from Polycystic ovary syndrome 13 women and the in vitro effects of BMP4, BMP6, and BMP7 in GCs steroidogenesis". European Journal of Endocrinology 168 (2013): 437-444.

27. Kristensen SG., et al. "Expression of TGF-beta superfamily growth factors, their receptors, the associated SMAD 3 antagonists in five size matched populations of preantral follicles from normal human ovaries". Molecular Human Reproduction 20 (2014): 293-308.

28. Nio-Kobayashi J., et al. "Bone morphogenetic proteins are mediators of luteolysis in the human corpus luteum". Endocrinology 156 (2015): 1494-1503.

29. Qiao J and Feng HL. "Extra and intra intraovarian factors in polycystic ovary syndrome: impact on oocyte maturation and embryo developmental competence". Human Reproduction Update 17 (2011): 17-33.

30. Regan SL., et al. "Dys regulation of granulosa bone morphogenetic protein receptor $1 \mathrm{~B}$ density is associated with reduced ovarian reserve and the age-related decline in human fertility". Molecular and Cellular Endocrinology 425 (2016): 84-93.

31. Childs AJ., et al. "BMP signaling in the human fetal ovary is developmentally regulated and promotes Primordial Germ Cell apoptosis". Stem Cells 28 (2010): 1368-1378.

32. Chang HM., et al. "Oocyte derived BMP15 but not GDF9 down regulate connexin 43 expression and decrease gap junction inter cellular communication activity in immortalized human granulosa cells". Molecular Human Reproduction 20 (2014): 373-383.

33. Eppig JJ. "Oocyte control of ovarian follicular development and function in mammals". Reproduction 122 (2001): 829-838.
34. Chang HM., et al. "Effects of Recombinant activin on steroidogenesis in human granulose-lutein cells". The Journal of Clinical Endocrinology and Metabolism 99 (2014b): E1922-1932.

35. Fang L., et al. "EGF like growth factors induce COX2 derived PGE2production through ERK1/2in human granulosa human granulosa cells". The Journal of Clinical Endocrinology and Metabolism 2013;98 (2013): 4932-4941.

36. Chang HM., et al. "BMP15 suppresses progesterone generation by down regulating StAR via ALK3 in human granulose-lutein cells". Molecular Endocrinology 27 (2013): 2093-2104.

37. Zhang H., et al. "BMP4 and BMP7 suppress StAR and progesterone production via ALK3 and SMAD 1/5/8- SMAD 4 in Human granulose-lutei n cells". Endocrinology 156 (2015): 42694280 .

38. Wigglesworth K., et al. "Transcriptomic diver sificatication of developing cumulus and mural cells in mouse ovarian follicles". Biology of Reproduction 92 (2015): 23.

39. Baranova NS., et al. "Incorporation of pentraxin 3 into hyaluronan matrices is tightly regulated and promotes matrix crosslinking". Journal of Biological Chemistry 289 (2014): 3048130498.

40. Wei LN., et al. "Reduced and delayed expression of GDF 9 and BMP 15 in ovarian tissue in women with Polycystic ovary syndrome". The Journal of Assisted Reproduction and Genetics 31 (2014): 1483-1490.

41. Asghari R., et al. "Alterations of TGFB1, GDF9and BMPR2 gene expression in preantral follicles of an estradio valerate induced Polycystic ovary syndrome mouse model can lead to anovulation, Polycystic morphology, obesity and absence of hyperandrogenism". Clinical and Experimental Reproductive Medicine 48.3 (2021): 245-254.

42. Simpson CM., et al. "Aberrant GDF9 expression and activation are associated with common human ovarian disorders". The Journal of Clinical Endocrinology and Metabolism 99 (2014): E615-624.

43. Chang HM., et al. "Effect of Growth Differentiation factor8 on steroidogenesis in human granulosa-lutein cells". Fertility and Sterility 2016b;105 (2016b): 520-528.

44. Chang HM., et al. "Activin A, B and AB decrease progesterone product ion by downregulating StAR in human granulose-lutein cells". Molecular and Cellular Endocrinology 412 (2015b): 290-301. 
45. Luo X., et al. "Bone morphogenetic protein inhibits Growth Differentiation factor induced cell signaling via up regulation of gremlin 2 expression in human granulosa-lutein cells". Reproductive Biology and Endocrinology 19 (2021): 173.

46. Bai L., et al. "The exploration of poor ovarian reserve-related risk factors:a potential role of Growth Differentiation factor8 in predicting ovarian response in IVF-ET patient". Frontiers in Endocrinology 12 (2021): 708089.

47. Gong Y., et al. "Age related decline in the expression of GDF 9 and BMP 15 genes in the folliclefluid and granulosa cells derived from poor ovarian responders". Journal of Ovarian Research 14 (2021): 1 .

48. Turathum B., et al. "The function of cumulus cells in oocyte growth and maturation and in subsequent ovulation and fertilization". Cells 10 (2021): 2292.

49. Magro-Lopez E and Munoz-Fernandes. "The role of BMP signaling in female reproductive system development and function". International Journal of Molecular Sciences 22 (2021): 11927.

50. Bai L., et al. "Aberrant elevations of GDF8impairs granulose cell glucose metabolism via up regulating SERPINE expression in patients with PCOS". Molecular Therapy - Nucleic Acids 23 (2021): 294-309.

\section{Assets from publication with us}

- Prompt Acknowledgement after receiving the article

- Thorough Double blinded peer review

- Rapid Publication

- Issue of Publication Certificate

- High visibility of your Published work

Website: $\underline{w w w}$.actascientific.com/

Submit Article: www.actascientific.com/submission.php

Email us: editor@actascientific.com

Contact us: +919182824667

Citation: Kulvinder Kochar Kaur., et al. "An Update on the Role of Bone Morphogenetic Protein and Growth Differentiation Factor on Follicular Generation in Human Menstrual Cycle with Clinical Impact on ART Success and PCOS Pathogenesis/Therapy-a Systematic Review". Acta Scientific Women's Health 4.2 (2022): 58-72. 